\title{
Extraction and Characterization of Cellulosic Nanowhisker obtained from Discarded Cotton Fibers
}

\author{
José Heriberto O. do Nascimento ${ }^{\mathrm{a}, *}$, Rodrigo F. Luz ${ }^{\mathrm{a}}$, Felipe M.F. Galvãoa, José Daniel \\ D. Melo ${ }^{\mathrm{b}}$, Fernando R. Oliveira ${ }^{\mathrm{a}}$, Rasiah Ladchumananandasivam ${ }^{\mathrm{a}}$, Andrea Zille ${ }^{\mathrm{c}}$ \\ ${ }^{a}$ Departamento de Engenharia Têxtil, Universidade Federal do Rio Grande do Norte UFRN, 59.072-970, Natal, Brazil \\ ${ }^{b}$ Departamento de Engenharia Mecanica, Universidade Federal do Rio Grande do Norte UFRN, 59.072-970, Natal, Brazil \\ ${ }^{c} 2 C 2 T$ - Centro de Ciência e Tecnologia Têxtil, Departamento de Engenharia Têxtil, Universidade do Minho, 4800-058 Guimarães, Portugal
}

\begin{abstract}
This study aimed to characterize cellulose nanowhiskers extracted from discarded cotton fibers. The nanocellulose was characterized by dynamic light scattering, zeta potential, Scanning Transmission Electron Microscopy (STEM), Atomic force microscopy (AFM) and X-ray powder diffraction (XRD). The nanocrystals are characterized by a rod-like morphology with sizes between $100 \mathrm{~nm}$ and $300 \mathrm{~nm}$. The acidic hydrolyses applied to the extract nanocrystals increased the crystallinity index from 72 to $86 \%$. The average length and diameter obtained were $98.1 \pm 4.7$ and $8.0 \pm 3.4 \mathrm{~nm}$, respectively. The aspect ratio of the fibers, around 12.3, was lower than the values usually reported in the literature. The dynamic light scattering results were in accordance with the nano crystal dimensions showing an average size of $235 \mathrm{~nm}$. Cellulose nanowhiskers obtained from cotton rejected by textile industry due to their abundance, low cost, high crystallinity, strength, low weight and biodegradability, are the ideal candidates for the processing of polymer nanocomposites.
\end{abstract}

(C) 2015 Elsevier Ltd. All rights reserved.

Selection and peer-review under responsibility of TEMA - Centre for Mechanical Technology and Automation.

Keywords: cotton; cellulose; nanowhiskes; nanocomposite; nanocrystal; nanocellulose

\section{Introduction}


Cellulose is a homopolymer of $\beta-1,4-d-$ glucose molecules linked in a linear chain that can be found in nature among various sources like plants, algae, marine creatures and bacteria. The single cotton fiber (thickness: $20-30 \mu \mathrm{m}$ ) consists of superfine fibrils having diameter in the range of nanometer. ${ }^{1}$ Cellulose, in its native state (termed cellulose-I) in the cell walls of plants and trees, forms a crystalline structure, whereby chains aggregate via hydrogen bonding. ${ }^{1}$ Two forms of crystalline cellulose are known to occur in plants namely cellulose I $\alpha$ and I $\beta$. All cellulose sources, however, are not completely crystalline. The presence of paracrystalline or amorphous cellulose is often significant, although it varies from species to species. The presence of this amorphous portion to cellulose makes the structure susceptible to acid hydrolysis, and the eventual breakdown into individual crystallites. ${ }^{2}$ Cellulose nanocrystals (CNC), sometimes referred as nanofibers, cellulose crystallites or simply crystals, are the crystalline domains of cellulose sources, with structure in nanoscale dimensions. ${ }^{3}$ Nanowhiskers are defined as the nanocellulose with diameters ranging from $2 \mathrm{~nm}$ to $20 \mathrm{~nm}$ and length ranging from $100 \mathrm{~nm}$ to $2.1 \mu \mathrm{m} .{ }^{4}$ These nanomaterials can be isolated from several natural fibers and are characterized by their low cost, abundance and biodegradability. ${ }^{5}$ When isolated, they can be applied in a wide range of applications in different fields, such as, engineered nanocomposites, construction materials, porous materials, intelligent or active package, functional surface among others. ${ }^{6}$ According to literature the main properties of nanocellulose materials are: biodegradability, biocompatibility, high strength, high surface area, moisture absorption, dimensional stability and chemical functionality. ${ }^{7,8}$ Cotton is one of the natural fibers with highest percentage of cellulose $(>95 \%)$ and it was widely used as source of nanocellulose 9 . However, the information about cotton nanowhiskers production methodologies and physico-chemical properties are scarce. ${ }^{10-12}$ Thus, the aim of this work was to extract and characterize the cellulose nanowhisker obtained from cotton rejected by textile industry. The physical and chemical properties of CNC obtained were studied by analysis of surface charge, crystal size dimension and morphology, crystallinity and chemical structure.

\section{Materials and methods}

\subsection{Cellulose nanowhiskers preparation}

A discarded $100 \%$ cotton fabric (textile industry residue) was used in this study. Cotton residues were milled and hydrolyzed by alkali treatment in a solution of $5 \%$ of $\mathrm{NaOH}$ at $80^{\circ} \mathrm{C}$. Then a bleaching process was applied in a bath composed by $3 \mathrm{~mL} / \mathrm{L}$ of $\mathrm{H}_{2} \mathrm{O}_{2} 200 \mathrm{~V}, 2 \mathrm{~mL} / \mathrm{L}$ of $\mathrm{NaOH} 38^{\circ} \mathrm{Be}, 1 \mathrm{~mL} / \mathrm{L}$ of stabilizer and $1 \mathrm{~mL} / \mathrm{L}$ of detergent. Finally, the method of acidic hydrolysis was applied with minor adaptations using a solution of $60 \% \mathrm{of}_{2} \mathrm{SO}_{4}$. At the end of process, the reaction was stopped by adding 10 times the volume of cold deionized water. The colloidal suspension was centrifuged for 20 minutes at $13.000 \mathrm{rpm}$ in a High-speed Refrigerated Centrifuge. The suspension obtained was ultra-sonicated to prevent agglomerates formation and dialyzed with tap water during $24 \mathrm{~h}$ until pH of 6-7 was reached. The process from centrifugation through dialysis was repeated three times.

\subsection{Dynamic Light Scattering (DLS)}

The particle size distribution (z-average-size) and the polydispersity index (PDI) of the silver nanoparticles were determined with a ZetaPlus instrument (Brookhaven Instruments Corporation). The samples were measured at a constant temperature of $25 \pm 1{ }^{\circ} \mathrm{C}$. The results were averaged over 30 runs. Each value was obtained by averaging measurements of three samples.

\subsection{Zeta Potential Measurements}

The z-potentials were determined in an electrophoretic lightscattering ZetaPlus apparatus (Brookhaven Instruments Corporation) to examine nanocellulose colloidal stability and charge. The electrophoretic determinations of $\mathrm{z}$ potentials were made in aqueous media at moderate electrolyte concentration. Each value was obtained by averaging measurements of three samples. 


\subsection{Scanning Transmission Electron Microscopy (STEM)}

STEM analysis of the nanowhiskers samples were carried out with an Ultra-high resolution Field Emission Gun Scanning Electron Microscopy (FEG-SEM), NOVA 200 Nano SEM, FEI Company. Secondary electron images were performed with an acceleration voltage between 5 and $10 \mathrm{kV}$. Backscattering Electron Images were realized with an acceleration voltage of $15 \mathrm{kV}$. The synthetized nanowhiskers suspension was dropped in copper grids with carbon film 400 meshes, $3 \mathrm{~mm}$ diameter. The average size was extrapolated measuring the average diameter based in 50 observations.

\subsection{X-ray powder diffraction (XRD)}

The crystalline phases of cellulose nanowhiskers were determined by using of X-ray diffractometer Schimadzu model: XRD 6000 with CuKa radiation in the range $20-70^{\circ}(\lambda=0.154 \mathrm{~nm})$.

\subsection{Atomic force microscopy (AFM)}

AFM experiments were performed in a tapping mode in air using a Multimode AFM from Agilent, model 5500, at room temperature. Silicon cantilevers (AppNano) with a constant force between 25 and $75 \mathrm{~N} \mathrm{~m}^{-1}$ and a resonance frequency of $339 \mathrm{kHz}$ were used. The PicoView 1.10 software version was used for the simultaneous recording of the topography, phase and amplitude magnitudes of the images.

\section{Results and discussion}

The amorphous regions of the cotton structure are the main target for the cleavage of the microfibrils into short monocrystals under acid hydrolysis due to their faster hydrolysis kinetics. The used method reveled to be a useful procedure for the preparation of highly crystalline particles of nanowhiskers. Under the used controlled conditions it was possible an efficient disruption of the amorphous regions surrounding and embedded within cellulose microfibrils leaving the microcrystalline segments intact. Since the geometrical characteristics of cellulose whiskers depend on the origin of cellulose microfibrils and acid hydrolysis process conditions such as time, temperature, and purity of materials, the morphological and physico-chemical characterization of nanowhiskers is extremely important for its potential uses as nanocomposite. ${ }^{13,14}$

The results of the zeta potential show that the nanocrystals are quite stables displaying an absolute value of $25.35 \pm 1.5 \mathrm{mV}$ (Fig. 1-a). A value of $25 \mathrm{mV}$ is widely reported in literature and it is considered the minimum value for stable nanocellulose dispersions. ${ }^{15}$ The particle size distribution of the nanosizer analysis is remarkably monodisperse showing a single sharp peak in intensity with sizes between 100 and $300 \mathrm{~nm}$ and an average size of $235 \mathrm{~nm}$ (Fig. 1-b). However, in the case of rod-like structure the dynamic light scattering display some limitations on the size estimation (Fig. 1-c). A more sensitive methodology like AFM will be necessary to confirm this data. The formation of a precipitate of insoluble clusters of nanocellulose particles was also observed. The analysis of the precipitate exhibits a wide peak indicating a high dispersion of sizes in micro range. 

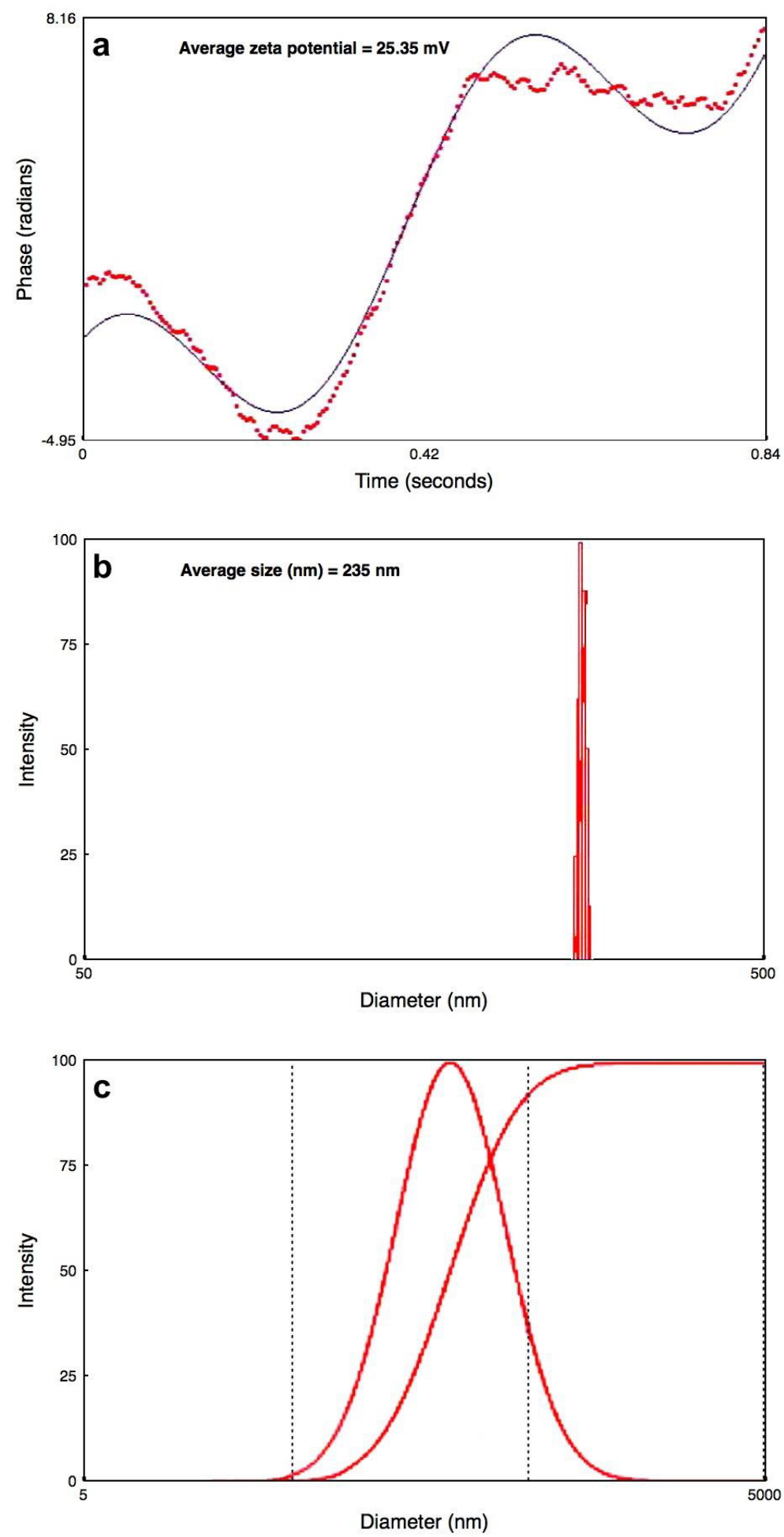

Fig.1. Nanosizer and Zeta potential results. 
The XRD patterns demonstrated the high crystallinity of the extracted cellulose nanowhiskers. The diffractograms of cellulose and nanowhiskers show the characteristic peaks of cellulose I at $22.5^{\circ}(200)$ and $34.5^{\circ}(040){ }^{16}$ The superimposed lattice peaks between $14.8^{\circ}(110)$ and $16.7^{\circ}(110)$ are better defined in the nanowhiskers cellulose than cellulose fibers. The higher crystallinity was confirmed by the calculation of the crystallinity index of the nanowhiskers (86\%) compared to the cellulose (72\%). An increase of $14 \%$ of crystallinity is in accordance with the available literature about the use of nanowhiskers as nanofiller for composites. ${ }^{9,17,18}$
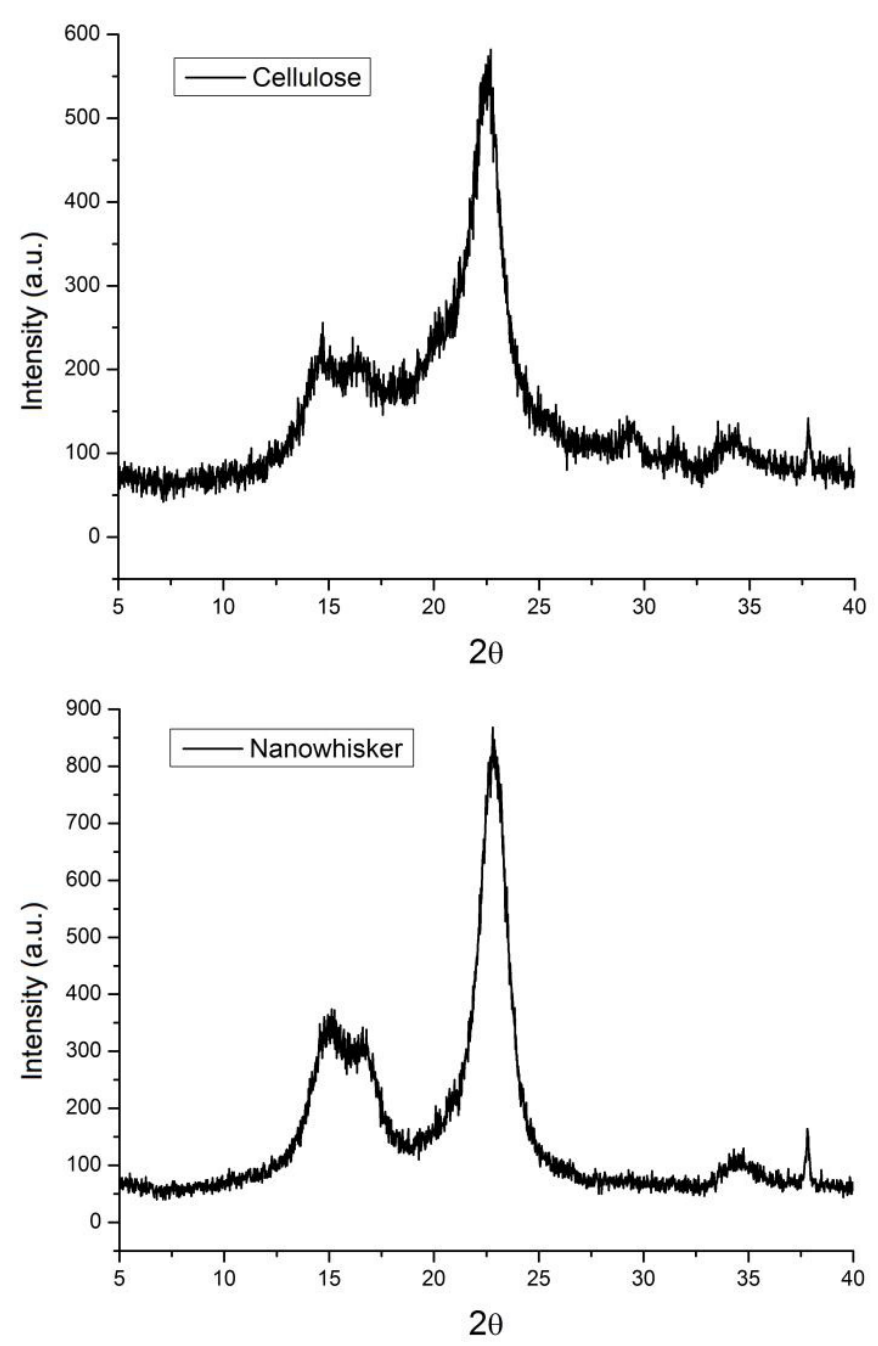

Fig 2. XRD measures of cellulose and nanowhiskers

The average length and diameter were estimated by AFM and STEM analysis with values of $98.1 \pm 4.7$ and $8.0 \pm 3.4 \mathrm{~nm}$, respectively (Fig. 3). The aspect ratio of the fibers, around 12.3, was lower than the values usually reported in literature. ${ }^{19,20}$ TEM picture reveals that cellulose nanowhiskers have a high surface area to volume ratio. This means that the surface could play a dominant role in the mechanical efficiency of stress transfer in a nanocomposite as well as in the ability to modify the surface chemistry. STEM picture reveal zone of agglomeration of nanocellulose with dispersed crystallites and individual crystals. 

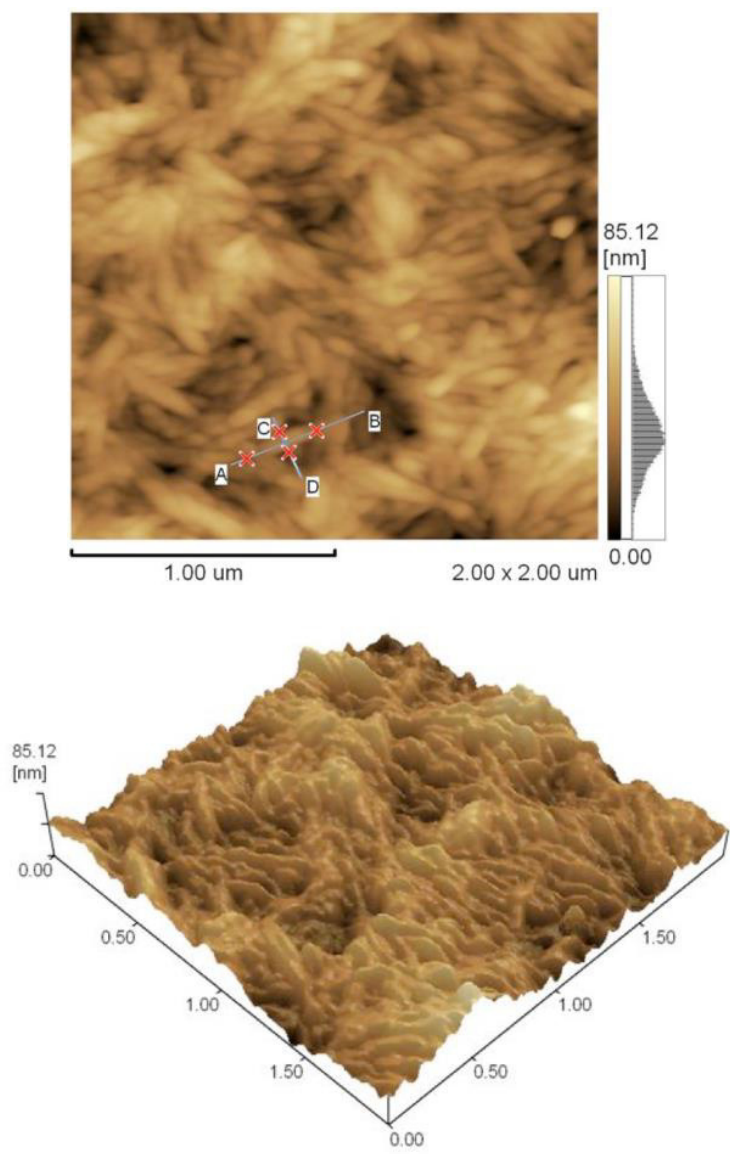

$2.00 \times 2.00[\mathrm{um}] \quad \mathrm{Z} 0.00-85.12[\mathrm{~nm}]$

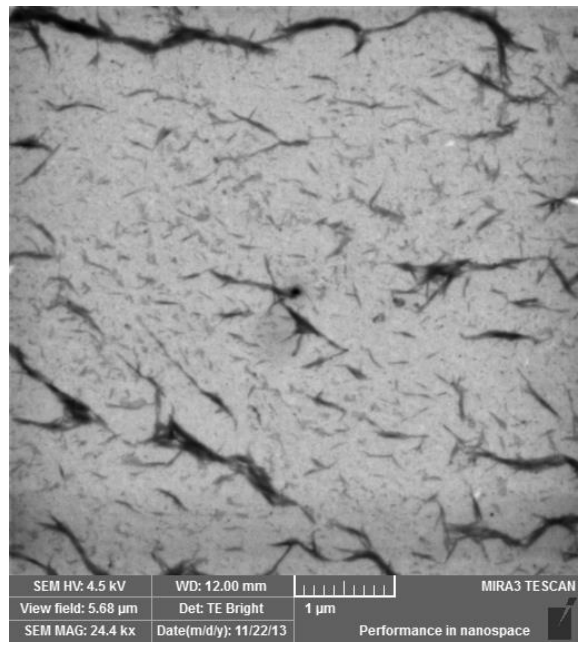

Fig.3. AFM topography images $(2 \times 2 \mu \mathrm{m})$ and STEM of the nanowhiskers. 


\section{Conclusion}

Cellulose nanowhiskers were successfully extracted by acid hydrolysis from cotton residues showing an aspect ratio of 12 and high crystallinity of $86 \%$. Nanocrystals sizes and dispersion was accurately measured by TEM, STEM and light scattering analysis. The results presented in this work suggest that the acidic route is an efficient alternative for obtaining monodisperse cellulose nanowhiskers from industrial cotton wastes.

\section{References}

1. $\quad$ Satyamurthy, P., et al., Carbohydrate Polymers (2011) 83 (1), 122

2. $\quad$ Eichhorn, S. J., Soft Matter (2011) 7 (2), 303

3. Azizi Samir, M. A. S., et al., Biomacromolecules (2005) 6 (2), 612

4. $\quad$ Rosa, M. F., et al., Carbohydrate Polymers (2010) 81 (1), 83

5. $\quad$ Eichhorn, S. J., Soft Matter (2011) 7 (2), 303

6. $\quad$ Siró, I., and Plackett, D., Cellulose (2010) 17 (3), 459

7. Hossain, K. M. Z., et al., Soft Matter (2012) 8 (48), 12099

8. $\quad$ Siqueira, G., et al., Biomacromolecules (2009) 10 (2), 425

9. $\quad$ Morais, J. P. S., et al., Carbohydrate Polymers (2013) 91 (1), 229

10. Adel, A. M., et al., Bioresource Technol (2010) 101 (12), 4446

11. Adel, A. M., et al., Carbohydrate Polymers (2011) 83 (2), 676

12. Gardner, D. J., et al., J Adhes Sci Technol (2008) 22 (5-6), 545

13. Bras, J., et al., Carbohydrate Polymers (2011) 84 (1), 211

14. Cao, X. W., et al., Carbohydrate Polymers (2012) 90 (2), 1075

15. Olszewska, A., et al., Soft Matter (2013) 9 (31), 7448

16. Elazzouzi-Hafraoui, S., et al., Biomacromolecules (2008) 9 (1), 57

17. Cherian, B. M., et al., Journal of Agricultural and Food Chemistry (2008) 56 (14), 5617

18. de Morais Teixeira, E., et al., Cellulose (2010) 17 (3), 595

19. Abdul Khalil, H. P. S., et al., Carbohydrate Polymers (2012) 87 (2), 963

20. Lavoine, N., et al., Carbohydrate Polymers (2012) 90 (2), 735 